Revista Ibero-Americana de Ciências Ambientais

Ibero-American Journal of Environmental Sciences

Edição Especial do Congresso de Gestão Ambiental do Baixo Amazonas

(CONGABA) Ago 2018 - v.9 - n.6

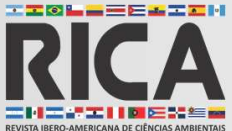

ISSN: 2179-6858

\title{
Diversidade de Odonata (Insecta) em igarapés na Reserva Extrativista Tapajós-Arapiuns (PA)
}

Estudos sobre estruturação de comunidades biológicas tem sido uma das principais abordagens em ecologia. Identificar as populações de determinada localidade nos possibilita propor estratégias direcionadas de conservação dos ecossistemas naturais que estão sendo modificados pelas ações humanas. Nesse contexto, foi realizado um levantamento da diversidade de espécies de Odonata adultos (Insecta) em corpos hídricos localizados na Reserva Extrativista Tapajós-Arapiuns (RESEX), Santarém/Aveiro (PA). Com uso de rede entomológica, foram amostrados adultos de Odonata em oito igarapés da RESEX. Os igarapés foram amostrados no mês de outubro de 2017. Cada igarapé foi amostrado uma vez em diferentes dias, totalizando oito oito coletas. Foram avaliados através de indices ecológicos a riqueza (Jackknife de primeira), diversidade (Shannon ( $H^{\prime}$ ) e Simpson (D)), dominância (Berger-Parker) e constância (Dajoz). Foram
coletados 230 indivíduos de Odonata, distribuídos em oito famílias, 17 gêneros e 19 espécies. A riqueza estimada de espécies foi de $25,96 \pm 2,40$ (média \pm desvio padrão), e a eficiência de coleta foi de $95 \%$. Encontramos uma considerável diversidade de espécies $\left(H^{\prime}=2,14 ; D=0,83\right)$. A espécie com maior dominância foi Chalcopteryx radians, com 62 indivíduos (26\%). Das 19 espécies coletadas, sete foram classificadas como constantes (36\%), seis como acessórias $(32 \%)$, e seis acidentais (32\%). A existência de uma grande diversidade de flora local possibilita para maioria dos indivíduos, um maior número de microhabitats, como no caso de Odonata. 0 fato de o levantamento ter sido realizado dentro de uma unidade de conservação, que possui atributos essenciais para manutenção das comunidades de Odonata, foi decisivo para existência da diversidade de espécies encontradas no levantamento. Ambientes estáveis, como no caso de áreas de preservação, podem proporcionar comunidades ricas de espécies. Essas características da RESEX acabaram possibilitando a dominância de espécies do gênero Chalcopteryx.

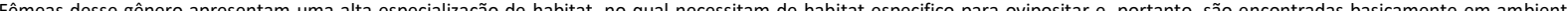
preservados. Considerando os resultados apresentados, é de grande importância manter a RESEX com sua integridade intacta e a realização de novos levantamentos da odonato, fauna com maior esforço amostral, para determinar de maneira mais consistente a diversidade local e possibilitar a utilização desses indivíduos em trabalhos de manejos da RESEX.

Palavras-chave: Amazônia; Distribuição; Libélula.

\section{Odonata (Insecta) diversity in streams in the Tapajós-Arapiuns Extractive Reserve (PA)}

\begin{abstract}
Studies on structuring biological communities have been one of the main approaches in ecology. Identifying the populations of a given locality enables us to propose directed strategies for Studies on structuring biological communities have been one of the main approaches in ecology. Identifying the populations of a given locality enables us to propose directed strategies for the conservation of natural ecosystems that are being modified by human actions. In this context, a survey was conducted on the diversity of adult Odonata (Insecta) species in water bodies
located in the Tapajós-Arapiuns Extractive Reserve (RESEX), Santarém/Aveiro (PA). Using an entomological network, adults from Odonata were sampled in eight RESEX streams. The streams were sampled in October 2017. Each stream was sampled once on different days, totaling eight collections. Ecological indexes were evaluated for richness (Jackknife prime), diversity (Shannon $\left(H^{\prime}\right)$ and Simpson (D)), dominance (Berger-Parker) and constancy (Dajoz). We collected 230 individuals from Odonata, distributed in eight families, 17 genera and 19 species. Estimated species richness was $25,96 \pm 2,40$ (mean \pm standard deviation), and collection efficiency was $95 \%$. We found a considerable diversity of species ( $H^{\prime}=2.14 ; D=0.83$ ). The most dominant species was Chalcopteryx radians, with 62 individuals (26\%). Of the 19 species collected, seven were classified as constant ( $36 \%)$, six as accessory (32\%), and six accidentals (32\%). The existence of a large diversity of local flora allows for most individuals a greater number of microhabitats, as in the case of Odonata. The fact that the survey was carried out within a conservation unit, which has essential attributes for maintaining Odonata communities, was decisive for the diversity of species found in the survey. Stable environments, such as preservation areas, can provide species (ich rich commities. The they require specific habitat integrity intact and to conduct new surveys of the odonate, fauna with greater sampling effort, to more consistently determine local diversity and enable the use of these individuals in management work of RESEX.
\end{abstract}

Keywords: Amazon; Distribution; Dragon-fly.

Topic: Conservação da Biodiversidade

Reviewed anonymously in the process of blind peer
Received: $11 / 08 / 2018$

Approved: $\mathbf{1 8 / 0 8 / 2 0 1 8}$
Everton Cruz da Silva

Universidade Federal do Oeste Pará, Brasil

http://lattes.cnpq.br/2090472105195603

evertonsilva856@gmail.com

\section{Tainã Rocha da Silva}

Universidade Federal do Oeste do Pará, Brasil

http://lattes.cnpq.br/8522407709031640

tainasilva.tr@gmail.com

Juliano de Sousa Ló

Universidade Federal do Oeste do Pará, Brasil

http://lattes.cnpq.br/6230650388307478

juliano-jslo@hotmail.com

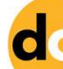

DOI: 10.6008/CBPC2179-6858.2018.006.0013
Amanda Karinne Amaral Lima

Universidade Federal do Oeste do Pará, Brasil

http://lattes.cnpq.br/2935663627563160

amanda.karinne96@gmail.com

José Max Barbosa Oliveira Junior (iD)

Universidade Federal do Oeste do Pará, Brasil

http://lattes.cnpq.br/1353014365045558

http://orcid.org/0000-0002-0689-205X

maxbio@hotmail.com
Referencing this:

SILVA, E. C.; SILVA, T. R.; LÓ, J. S.; LIMA, A. K. A.; OLIVEIRA JUNIOR, J. M. B.. Diversidade de Odonata (Insecta) em igarapés na Reserva Extrativista Tapajós-Arapiuns (PA). Revista Ibero Americana de Ciências Ambientais, v.9, n.6, p.109-119, 2018. DOI:

http://doi.org/10.6008/CBPC2179-6858.2018.006.0013 


\section{INTRODUÇÃO}

Por apresentar enorme riqueza de espécies, os insetos aquáticos possuem grandes aplicações em estudos biológicos de conservação. Ainda que representem significativa parcela da biodiversidade exercendo grandes influências sobre as dinâmicas dos ecossistemas tropicais, esses animais ainda não são utilizados com frequência na escolha de áreas prioritárias para instalação de unidades de conservação especialmente em território brasileiro (MARCO et al., 2004).

Devido à facilidade de coleta e sua grande sensibilidade às alterações do meio, a utilização de invertebrados aquáticos como bioindicadores nos sistemas de avaliação de impactos sobre o meio ambiente nos dias atuais, possui grande relevância (GOULART et al., 2003). Um dos representantes de invertebrados bastante utilizados neste tipo de estudo é a ordem Odonata, por serem predadoras generalistas de topo de cadeia trófica de lagos e riachos, isso faz com que qualquer modificação nas características do meio ambiente afete diretamente a dinâmica desses animais (CARVALHO et al., 1998).

Pertencente à classe Insecta, a ordem Odonata é conhecida no cotidiano como libélula, lava-bunda, lavadeira, cavalo-de-judeu, zig-zag e jacinta (SOUZA et al., 2007). Essa ordem é dividida em três subordens: Anisoptera, Zygoptera e Anisozygoptera, mas apenas as duas primeiras ocorrem no Brasil, juntas formam um grupo de aproximadamente sete mil espécies (MIGUEL et al., 2017). Devido suas exigências ecofisiológicas esses animais apresentam padrões de comportamentos classificados como: 'Flier', onde os indivíduos permanecem a maior parte do tempo em voo, e outros chamados de 'Percher', onde eles ficam em oscilações entre voos rápidos e com maior parte do tempo empoleirados (COBERT et al., 2008).

Para os Odonata, a quantidade de tempo que uma espécie fica diretamente exposta ao sol (insolação) pode afetar o seu comportamento reprodutivo e limitar o tempo para defesa territorial durante o dia (MARCO et al., 2005). Estes fatores são relevantes principalmente para os indivíduos ectotérmicos, uma vez que dependem dos atributos termais do hábitat. Os 'Flier' apresentam tamanho corporal maior e produzem calor metabólico, com isso podem permanecer mais tempo desempenhando suas atividades. Já os 'Percher' são, em geral, menores dos que os 'Flier', e podem ser divididos em maiores e menores, sendo que os primeiros são heliotérmicos e utilizam a luz solar como fonte externa de calor para aquecer seus corpos e desempenhar suas atividades diárias. Já os menores são conformadores termais, e se aquecem através do processo de convecção. Desta forma, dependem da temperatura externa para aquecerem seus corpos e iniciarem suas atividades (CORBET, 1999).

Tendo como base as informações expostas, é de grande importância a realização de uma avaliação do padrão de estruturação das comunidades de adultos de Odonata em corpos hídricos localizados em áreas de proteção ambiental. Diante disso, o presente trabalho objetivou realizar um levantamento da diversidade de espécies de Odonata adultos (Insecta) em igarapés localizados na RESEX Tapajós-Arapiuns nos municípios de Santarém e Aveiro, (PA). 


\section{MATERIAIS E MÉTODOS}

\section{Área de Estudo}

O estudo foi desenvolvido na Reserva Extrativista Tapajós-Arapiuns (RESEX), localizada nos Municípios de Santarém e Aveiro, na região oeste do Estado do Pará, com uma área de 647.610ha, entre as coordenadas geográficas $02^{\circ} 20^{\prime}$ a $03^{\circ} 40^{\prime}$ Sul, e $55^{\circ} 00^{\prime}$ a $56^{\circ} 00^{\prime}$ Oeste. A RESEX foi criada pelo Decreto Presidencial publicado em 06 de novembro de 1998 (BRASIL, 1998). O acesso a essa reserva pode ser feito por via fluvial, a partir de Santarém e Itaituba, pelos Rios Tapajós e Arapiuns (CARVALHO JUNIOR, 2008). O clima da região é tropical úmido (tipo Ami de Köeppen), com temperatura média anual de 26 a $27^{\circ} \mathrm{C}$, umidade relativa do ar em torno de $86 \%$ e precipitação anual variando entre 1.287 e $2.538 \mathrm{~mm}$, sendo fortemente sazonal.

A estação chuvosa vai de dezembro a julho, com médias mensais variando entre 170 e $300 \mathrm{~mm}$, e a estação seca vai de agosto a novembro, com médias mensais inferiores a $60 \mathrm{~mm}$. 0 clima é fortemente influenciado pelo fenômeno El Niño, e quando este ocorre, o período de seca é mais longo e intenso, o que deixa a vegetação extremamente vulnerável a incêndios florestais (BARLOW et al., 2004).

\section{Coleta de Dados: amostragem de Odonata}

Foram amostrados oito igarapés no mês de outubro de 2017 (período de estiagem). Cada igarapé foi amostrado uma vez em dias alternados, totalizando oito coletas distintas, utilizando a metodologia de varredura em áreas fixas, na qual foram demarcados 100m (trechos) em cada igarapé, subdivididos em 20 transectos de cinco metros de comprimento. O tempo de permanência em cada transecto foi de cinco minutos (MARCO et al., 2002; MARCO, 1998).

Para coleta dos indivíduos adultos, utilizou-se uma rede entomológica $(40 \mathrm{~cm}$ de diâmetro, $65 \mathrm{~cm}$ de profundidade e cabo de alumínio com $90 \mathrm{~cm}$ de comprimento). Os indivíduos capturados foram fixados e acondicionados em envelopes entomológicos. Para identificação, foram utilizadas chaves de identificação taxonômica especializadas (GARRISON et al., 2010). Após a identificação, os espécimes foram depositados no laboratório Multidisciplinar de Gestão ambiental da Universidade Federal do Oeste do Pará, Santarém (PA).

\section{Análise dos dados}

Com intuito de estimar a riqueza de espécies nos igarapés, foi usado o estimador não paramétrico Jackknife de primeira ordem (COLWELL et al., 1994), controlando o esforço de amostragem. A eficiência de amostragem de espécies de Odonata foi verificada por meio de curvas de acumulação de espécies, utilizando o mesmo estimador com 1.000 aleatorizações, baseado no número de segmentos (COLWELL et al., 2004).

O cálculo da diversidade foi realizado utilizando-se o índice de Shannon-Wiener $\left(\mathrm{H}^{\prime}\right)$, sendo a riqueza e seus intervalos de confiança (95\%) obtidos também pelo estimador analítico Jackknife, e o índice de Simpson para medir a equitabilidade, que avalia probabilidade de dois indivíduos escolhidos ao acaso 
pertencerem à mesma espécie, esse índice varia de 0 a 1 e quanto mais próximo de um, maior a diversidade de espécies.

Foi mensurada a dominância através do índice de Berger-Parker, esse índice considera a maior proporção da espécie com maior número de indivíduos. É calculada através da equação $d=\frac{N m a x}{N T}$, onde: d é a dominância; Nmax é o número de indivíduos da espécie mais abundante; e NT é o número total de indivíduos na amostra.

O índice de constância de Dajoz (1973) foi calculado para cada táxon ocorrente nos pontos de coleta, a fim de observar a porcentagem de ocorrência de cada espécie nos pontos estudados, sendo considerados táxon constantes quando ocorrência $>50 \%$, acessório entre 49 a $25 \%$ e acidental quando verificada ocorrência abaixo de $25 \%$. As estimativas de riqueza de espécies foram calculadas no programa EstimateS (Statistical Estimation of Species Richness and Shared Species from Samples) versão 7.5.0, e as demais análises foram realizadas pelas rotinas do programa R DEVELOPMENT CORE TEAM, usando o pacote Vegan (CÁCERES et al., 2009).

\section{DISCUSSÃO TEÓRICA}

Foram coletados 230 indivíduos de Odonata, distribuídos em oito famílias, 17 gêneros e 19 espécies. A subordem Zygoptera apresentou 216 indivíduos, distribuídos em seis famílias e 15 espécies; já a subordem Anisoptera apresentou 14 indivíduos distribuídos em duas famílias e cinco espécies. As famílias mais abundantes foram Calopterygidae com $(n=65)$, seguida por Polythoridae $(n=62)$ e Coenagrionidae $(n=58)$ (figura 1). Os gêneros mais representativos foram: Chalcopteryx (uma espécie e 62 indivíduos), Mnesarete (uma espécie e 55 indivíduos) e Epipleoneura (uma espécie e 43 indivíduos).

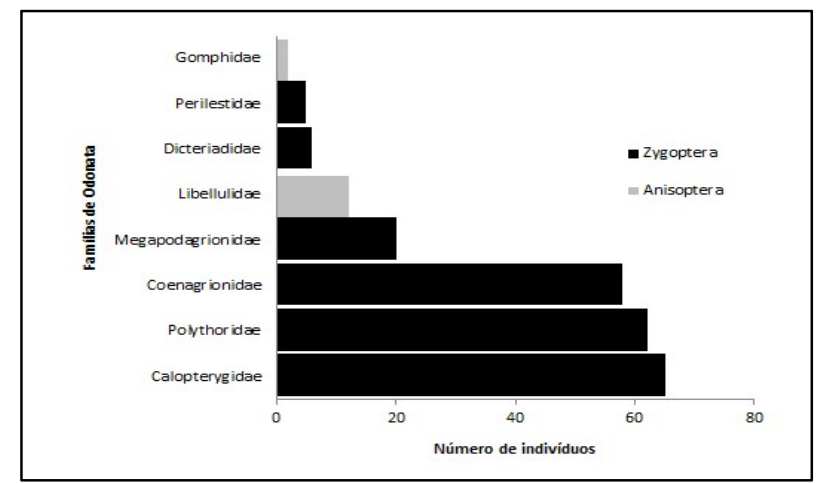

Figura 1: Abundância das famílias da ordem Odonata (Insecta) ao longo dos oito igarapés de amostragem na RESEX Tapajós-Arapiuns, Santarém/Aveiro (PA).

As espécies com maior abundância foram Chalcopteryx radians (62 indivíduos), seguida por Mnesarete aenea (53 indivíduos) e Epipleoneura cappilliformis (43 indivíduos) (tabela 1). A riqueza de espécies estimada pelo Jackknife foi de $25,96 \pm 2,40$ (média \pm desvio padrão) e a eficiência de coleta (Jackknife 1/Rarefação) foi de 95\%, conforme se pode ver através da figura 2. 
Tabela 1: Abundância de espécies de Odonata (Insecta) em oito igarapés amostrados na RESEX Tapajós-Arapiuns, Santarém/Aveiro (PA).

\begin{tabular}{|c|c|c|c|c|c|c|c|c|c|}
\hline \multirow{2}{*}{ Subordens/famílias/espécies } & \multicolumn{8}{|c|}{ IGARAPÉS } & \multirow{2}{*}{ Total } \\
\hline & IG.1 & IG.2 & IG.3 & IG.4 & IG.5 & IG.6 & IG.7 & IG.8 & \\
\hline \multicolumn{10}{|l|}{ ANISOPTERA } \\
\hline \multicolumn{10}{|l|}{ Gomphidae } \\
\hline Agriogomphus spp. (Belle, 1966) & 0 & 0 & 0 & 0 & 1 & 0 & 0 & 0 & 1 \\
\hline Zonophora calippus (Selys, 1869) & 1 & 0 & 0 & 0 & 0 & 0 & 0 & 0 & 1 \\
\hline \multicolumn{10}{|l|}{ Libellulidae } \\
\hline Erythrodiplax fusca (Rambur, 1842) & 2 & 0 & 0 & 0 & 0 & 0 & 1 & 0 & 3 \\
\hline Fylgia amazonica (Kirby, 1889) & 2 & 0 & 0 & 0 & 0 & 1 & 0 & 0 & 3 \\
\hline Oligoclada amphiname (Ris, 1919) & 0 & 0 & 1 & 0 & 3 & 2 & 0 & 0 & 6 \\
\hline \multicolumn{10}{|l|}{ ZYGOPTERA } \\
\hline \multicolumn{10}{|l|}{ Calopterygidae } \\
\hline Hetearina indeprensa (Garrison, 1990) & 0 & 2 & 0 & 5 & 3 & 0 & 0 & 0 & 10 \\
\hline Mnesarete aenea (Selys, 1853) & 11 & 7 & 1 & 6 & 8 & 5 & 12 & 5 & 53 \\
\hline Mnesarete spp. & 0 & 0 & 0 & 1 & 0 & 0 & 0 & 0 & 1 \\
\hline \multicolumn{10}{|l|}{ Coenagrionidae } \\
\hline Argia sp. (Selys, 1865) & 0 & 4 & 1 & 2 & 1 & 2 & 0 & 1 & 11 \\
\hline Epipleoneura cappilliformis (Ris, 1914) & 5 & 2 & 6 & 9 & 5 & 2 & 5 & 9 & 43 \\
\hline Neoneura denticulata (Williamson, 1917) & 0 & 0 & 0 & 0 & 1 & 0 & 0 & 0 & 1 \\
\hline Phasmoneura exigua (Selys 1886) & 0 & 0 & 0 & 0 & 2 & 2 & 0 & 0 & 3 \\
\hline \multicolumn{10}{|l|}{ Dicteriadidae } \\
\hline Dicterias atrosanguinea (Selys, 1853) & 0 & 0 & 0 & 0 & 0 & 0 & 0 & 2 & 2 \\
\hline Heliocharis amazona (Selys, 1853) & 1 & 0 & 0 & 0 & 0 & 1 & 1 & 1 & 4 \\
\hline \multicolumn{10}{|l|}{ Megapodagrionidae } \\
\hline Heteragrion bariai (Marmels, 1989) & 1 & 0 & 2 & 0 & 1 & 1 & 0 & 1 & 6 \\
\hline Oxystigma spp. (Geijskes,1976) & 0 & 0 & 0 & 0 & 0 & 0 & 1 & 0 & 1 \\
\hline Oxystigma williamsoni (Geijskes, 1976) & 1 & 0 & 0 & 0 & 2 & 0 & 5 & 6 & 13 \\
\hline \multicolumn{10}{|l|}{ Perilestidae } \\
\hline Perissolestes cornutus (Watson, 1991) & 0 & 0 & 0 & 0 & 0 & 0 & 0 & 5 & 5 \\
\hline \multicolumn{10}{|l|}{ Polythoridae } \\
\hline Chalcopteryx radians (Ris, 1914) & 27 & 19 & 0 & 3 & 4 & 1 & 8 & 0 & 62 \\
\hline Total geral & 51 & 34 & 10 & 26 & 29 & 17 & 33 & 30 & 230 \\
\hline
\end{tabular}

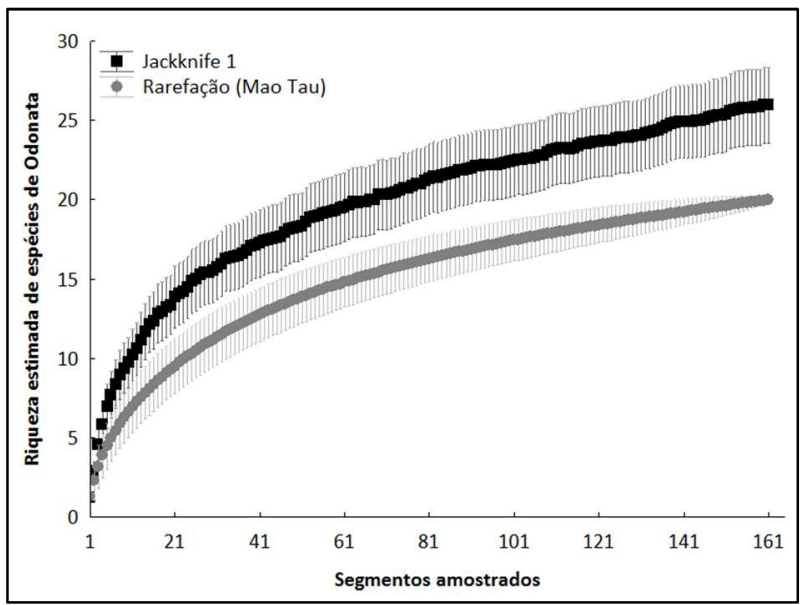

Figura 2: Curva de acumulação de espécies (Jackknife de primeira ordem e Mao Tau; média \pm intervalo de confiança) de Odonata (Insecta) coletadas em Igarapés da RESEX Tapajós-Arapiuns, Santarém/Aveiro, (PA).

Os resultados encontrados são semelhantes aos obtidos na reserva Ducke, que indicou 19 espécies (JUEN et al., 2011), em Serra das Torres, Sul do Estado do Espírito Santo, que identificou a ocorrência de 22 espécies (SILVA et al., 2007) e a do Parque Estadual de Itapuã, Porto Alegre, com 23 espécies (MARINS et al., 2005), e maior do que o encontrado no município de Colatina (ES) com nove espécies (BOTI et al., 2007). A curva de rarefação da reserva Tapajós-Arapiuns, obtida do total de amostra, ainda se encontra em processo de estabilização, o que indica que as coletas realizadas não foram suficientes para capturar toda a riqueza local. 
Por ser uma unidade de conservação, a RESEX possui forte proteção de órgãos ambientais, e conta com a fiscalização de parcerias entre o governo e os moradores, mantendo sua biodiversidade quase intacta (CARVALHO et al., 2008). A existência de uma grande diversidade de flora local possibilita para maioria dos indivíduos a existências de um maior número microhabitats, como no caso de Odonata (JANKOWSKI et al., 2009). Isso permite uma maior disponibilidade de recursos e consequentemente uma maior quantidade de espécies (OLIVEIRA-JUNIOR et al., 2013).

Em futuros levantamentos nessa localidade pode haver um aumento no número de espécies. Outro fator importante a ser levado em consideração é que a reserva Tapajós-Arapiuns possui uma área de 647.610ha, e as coletas foram realizadas em cada corpo hídrico em um transecto de $100 \mathrm{~m}$, totalizando $800 \mathrm{~m}$ de área amostral, o que demonstra que o esforço amostral pode não ter sido suficiente para caracterizar toda a riqueza de espécies existente na reserva.

Encontramos uma considerável diversidade de espécies $\left(H^{\prime}=2,14\right)$, semelhante ao que foi registrado no levantamento realizado na Estação Ecológica do Seridó no município de Serra Negra (RN) $\left(H^{\prime}=2,29\right)$ (FERREIRA et al., 2009), em áreas preservadas do Vale do Taquari $\left(H^{\prime}=2,46\right)$ (CONSATTI et al., 2014). O valor do índice de Simpson $(D=0,83)$ corrobora com resultado apresentado por Shannon indicando uma alta diversidade de espécies no local. Segundo Begon (2007), ambientes mais estáveis, como no caso de áreas de preservação, podem proporcionar comunidades mais ricas de espécies.

Atributos como riqueza, distribuição e diversidade são influenciados, tanto pela qualidade dos recursos disponíveis, quanto pela qualidade do ambiente (HURYN et al., 2000). Para Lomolino et al. (2004), as espécies são constantemente influenciadas pelas características físicas do ambiente como: temperatura, umidade do ar, parâmetros físicos da água, pluviosidade e luminosidade do ambiente. Essas características agem como filtros que vão definir diversidade, abundância e distribuição espacial desses indivíduos (URILCH, 2008).

O fato de o levantamento ter sido realizado dentro de uma unidade de conservação, que possui atributos essenciais para reprodução das comunidades de Odonata, pode ter sido decisivo para o resultado apresentado pelos índices de diversidade. Em um recente trabalho realizado por Monteiro-Junior et al. (2016) na Reserva de Caxiuanã, a unidade de conservação foi fundamental na manutenção da diversidade de Odonata, Monteiro-Junior et al. (2016) também chama atenção para importância das áreas adjacentes que também contribuem para esse processo, desde que sejam abrangidas condições ecológicas adequadas. A alta diversidade de espécies de Odonata pode ter sido um reflexo das boas condições de integridade dos corpos hídricos estudados e da presença de vegetação ciliar.

A grande quantidade de microhabitats disponibilizados pelas matas ciliares dos igarapés é importante para manutenção das espécies de Odonata, tendo em vista que as características do ambiente influenciam no padrão de distribuição das mesmas (DALZOCHIO et al., 2011). A espécie com maior dominância foi Chalcopteryx radians com 62 indivíduos (26\%), conforme trazido pela tabela 2.

A elevada abundância de indivíduos dessa espécie reflete o equilíbrio desse ecossistema estudado. Fêmeas de Chalcopteryx apresentam uma alta especilização de habitat, no qual necessitam de habitat 
especifico para ovipositar e, portanto, são encontradas basicamente em ambientes preservados (OLIVEIRAJUNIOR et al., 2015); em caso de distúrbio ambiental, devido suas exigências ecofisiológicas, uma redução no número de indivíduos pode ser observada (CLAUSNITZER, 2003). Por ser um gênero restrito a florestas primárias, é portanto, considerado um bom bioindicador da integridade de ecossistemas aquáticos (GUILHERMO-FERREIRA et al., 2014; MONTEIRO-JÚNIOR, 2012).

Quanto à frequência de ocorrência das espécies, observou-se que das 19 espécies coletadas, sete foram classificadas como constante (36\%): Argia sp., Chalcopteryx radians, Epipleoneura cappilliformis, Heliocharis amazona, Heteragrion bariai, Mnesarete aenea e Oxystigma williamson, seis como acessórias (32\%): Agriogomphus spp., Erythrodiplax fusca, Fylgia amazônica, Hetearina indeprensa, Oligoclada amphiname e Phasmoneura exígua, e seis acidentais (32\%): Dicterias atrosanguinea, Mnesarete spp., Neoneura denticulata, Oxystigma spp., Perissolestes cornutus e Zonophora calippus, conforme ilustrado pela figura 3 e de acordo com o proposto pela tabela 2 .

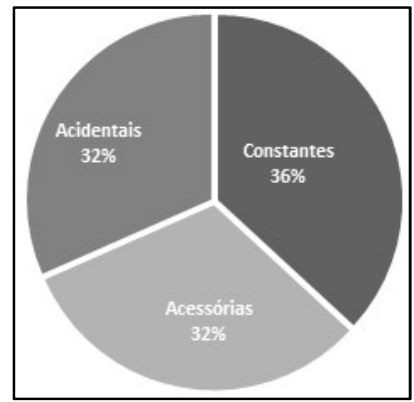

Figura 3: Representação percentual da Constância das espécies da ordem Odonata (Insecta) amostradas na RESEX Tapajós-Arapiuns, Santarém/Aveiro (PA).

Tabela 2: Número de indivíduos (N), Frequência relativa de ocorrência (\%) das espécies de Odonata (Insecta) na RESEX Tapajós-Arapiuns, Santarém/Aveiro (PA), classificadas como: Constantes (CT), Acessórias (AC) e acidentais (AD).

\begin{tabular}{|l|c|c|c|c|c|}
\hline \multicolumn{1}{|c|}{ Espécies } & $\mathbf{N}$ & Frequência (\%) & Constância & Dominância (\%) \\
\hline Agriogomphus spp. & 1 & 12,5 & AC & 0,43 \\
\hline Argia sp. & 11 & 75 & CT & 4,78 \\
\hline Chalcopteryx radians & 62 & 75 & CT & 26,96 \\
\hline Dicterias atrosanguinea & 2 & 12,5 & AD & 18,87 \\
\hline Epipleoneura cappilliformis & 43 & 100 & CT & 1,30 \\
\hline Erythrodiplax fusca & 3 & 25 & AC & 1,30 \\
\hline Fylgia amazônica & 3 & 25 & AC & 1,74 \\
\hline Heliocharis amazona & 4 & 50 & CT & 4,35 \\
\hline Hetearina indeprensa & 10 & 37,5 & AC & 2,61 \\
\hline Heteragrion bariai & 6 & 62,5 & CT & 23,04 \\
\hline Mnesarete aenea & 53 & 87,5 & CT & 0,43 \\
\hline Mnesarete spp. & 1 & 12,5 & AD & 0,43 \\
\hline Neoneura denticulata & 1 & 12,5 & AD & 2,61 \\
\hline Oligoclada amphiname & 6 & 37,5 & AC & 0,43 \\
\hline Oxystigma spp. & 1 & 12,5 & AD & 5,65 \\
\hline Oxystigma williamsoni & 13 & 50 & AD & 2,17 \\
\hline Perissoleste cornutus & 5 & 3 & 25 & AC & 1,30 \\
\hline Phasmoneura exígua & 1 & 12,5 & AD & 0,43 \\
\hline Zonophora calippus & & & \\
\hline
\end{tabular}

As espécies Argia sp., Epipleoneura cappilliformis, Heteragrion bariai, e Oxystigma williamsoni pertencem as famílias Coenagrionidae e Megapodagrionidae, que são bioindicadoras de ambientes preservados. Indivíduos do gênero Heteragrion geralmente habitam em riachos dentro das florestas (GARRISON et al., 2010), e segundo Schmidt (1985), são considerados bons indicadores de qualidade da água. 
Além disso, segundo Machado et al. (1988), Heteragrion é um gênero muito sensível à deterioração ambiental.

A presença de indivíduos desse gênero dentro da unidade de conservação, de forma constante é um forte argumento para bioindicação de que a qualidade da água e de que o ambiente em geral está em boas condições de preservação. Espécies do gênero Heteragrion são citadas por Machado et al. (1991) na Reserva Biológica de Maracá, em Roraima, e também por Monteiro-Júnior (2012) em seu trabalho na Reserva Florestal Adolpho Ducke, em Manaus.

As espécies da família Coenagrionidae também são citadas em trabalhos como de Côrtes (2011) na Estação Ecológica Serra Geral do Tocantins (EESGT), por Hanauer et al. (2014) no Vale do Taquari Rio Grande do Sul. Espécies do gênero Argia tem preferência por ambientes mais íntegros, com presença de vegetação primária e de macrófitas para realização da ovoposição, comuns de serem encontradas e capturadas em locais preservados devido suas exigências ecofisiológicas e seu comportamento territorialista (MARTINS, 2004).

Outra espécie que apareceu no levantamento na categoria constante foi Heliocharis amazona que, segundo Ferreira-Peruquetti et al. (2003), devido à sua alta sensibilidade as mudanças no ambiente, reflete o bom estado de estabilidade local. Essa espécie é a única do gênero e possui registros nas Américas com início no nordeste da Argentina até a Guiana Francesa.

As espécies Oligoclada amphiname, Erythrodiplax fusca, Fylgia amazônica pertencem a família Libellulidae que foram classificadas na categoria acessória. A família Libellulidae (Anisoptera) apresenta uma alta diversidade nas regiões tropicais, de onde provavelmente deve ter sido originada (DANTAS, 2017). Os Anisoptera possuem uma ampla distribuição devido ao seu tamanho corporal, o que aumenta a vagilidade e consequentemente sua dispersão e distribuição (DALZOCHIO, 2011). Isso possibilita a presença desses animais em diversos ambientes.

Geralmente, esses indivíduos têm preferência por ambientes abertos devido suas atividades termorreguladoras, não sendo comum a presença em igarapés com os níveis de conservação que a RESEX apresenta. Um reflexo das exigências ecofisiológicas dessa subordem é o pouco número de espécimes coletados, onde do total de indivíduos amostrados $(\mathrm{N}=230)$ apenas 14 pertencem à subordem Anisoptera. $\mathrm{A}$ espécie Hetearina indeprensa pertence à família Calopterygidae, e como a maior parte da subordem Zygoptera tem preferências por locais preservados, sendo encontradas na maior parte das vezes em riachos de vegetação densa (SILVA, 2007).

A eficiente exploração da área amostral permitiu à coleta de espécimes rara (categorizadas como acidentais), que devido seu baixo grau de tolerância a distúrbios ambientais, sua presença acaba se restringindo a ambientes estáveis, como indivíduos da família Dicteriadidae. A espécie Dicterias atrosanguinea é muito sensível a perturbações ambientais, sendo encontrada geralmente em ambientes totalmente preservados. Essa espécie está na lista brasileira de espécies ameaçadas de extinção (MONTEIROJUNIOR et al., 2016). 
Outra espécie foi Neoneura denticulata, pertencente à família Coenagrionidae. Ela possui dependência de vegetação densa apresentando maior abundância em ambientes preservados (OLIVEIRAJUNIOR et al., 2013). Espécies da familia Coenagrionidae, por serem menores, podem ser mais sensíveis às variações ambientais por restrições ecofisiológicas, podendo ser consideradas como umas das familias da ordem Odonata de foco prioritário para as estratégias de conservação de ambientes áquaticos (JUEN et al., 2011).

Perissolestes cornutus pertence à família Perissolestes e à subordem Zygoptera, sendo categorizada nesse levantamento como acidental, aparece como não preocupante na lista brasileira de espécies. Os dados em relação a essa espécie ainda são escassos devido à pouca frequência de ocorrência em estudos. Os indivíduos da família Perissolestes geralmente habitam florestas, principalmente florestas tropicais. Eles são encontrados em lugares escuros, empoleirados em ramos de árvores perto de pequenos riachos com dominância de árvores no dossel (SORIANO, 1978). A espécie Zonophora calippus, da família Gomphidae, é uma das representantes da subordem Anisoptera.

Os organismos dessa família possuem grande tamanho corporal e excelente capacidade de voo, o que the permite uma ampla distribuição, devido seu comportamento de grande vagilidade (CORBET, 1999). Essa característica possibilita uma maior dispersão dos indivíduos em uma grande quantidade de ambientes distintos (DALZOCHIO, 2011). O estado de conservação da RESEX foi de fundamental importância para grande diversidade de espécies encontrada. A presença da vegetação ripária propiciou um elevado número de espécies de Zygoptera devido às mesmas dependerem de áreas cobertas por vegetação densa (CORBET, 1999). Um fator com maior influência nas diferenças estruturais das comunidades de Odonata dos corpos hídricos são as matas ciliares (OLIVEIRA-JUNIOR et al., 2017).

Modificações negativas sobre esse importante componente natural, pode resultar em vários problemas ambientais, como extinção de espécies, quantidade de luz que entra no ambiente, modificação no clima, erosão do solo e até mesmo o assoreamento do curso da água (FERREIRA et al., 2004). Por isso, é de suma importância a manutenção da conservação da RESEX e a realização de novos levantamentos de estruturação de comunidades biológicas.

\section{CONCLUSÕES}

Existe uma considerável diversidade de espécies na RESEX Tapajós-Arapiuns, isso é um reflexo das boas condições de conservação dos corpos hídricos, com contribuição das áreas adjacentes. Esses conjuntos de fatores influenciam na manutenção da diversidade local de Odonata. Considerando os resultados apresentados, é de grande importância manter a RESEX com sua integridade intacta e a realização de novos levantamentos da odonatofauna com maior esforço amostral, para determinar de maneira mais consistente a diversidade local e possibilitar a utilização desses indivíduos em trabalhos de manejos da RESEX.

\section{REFERÊNCIAS}

BARLOW, J.; PERES, C. A.. Avifaunal responses to single and recurrent wildfires in Amazonian forests. Ecological
Applications, Washington, v.14, n.5, p.1358-1373, 2004.

DOI: https://doi.org/10.1890/03-5077 
BEGON, M.; TOWNSEND, C. R.; HARPER, J. L.. Ecologia: de indivíduos e ecossistemas. 4 ed. Porto Alegre: Artmed, 2007.

BOTI, J. B.; TOLEDO L. O.; HADDADE, I. R.; BOTI, D. R.; OLIVEIRA, B. R.. Libélulas (Odonata: Insecta) como indicadoras de poluição do córrego São Silvano, Colatina, ES. In: CONGRESSO DE ECOLOGIA DO BRASIL, 7. Anais. Caxambu: SEB, 2007.

BRASIL. Decreto de 06 de novembro de 1998. Institui a criação nos municípios de Santarém e Aveiro, no Estado do Pará, a Reserva Extrativista Tapajós-Arapiuns. Brasília: DOU, 1998.

CÁCERES, M.; LEGENDRE, P.. Associations between species and groups of sites: indices and statistical inference. Ecology, v.90, n.12, p.3566-3574, 2009. DOI: https://doi.org/10.1890/08-1823.1

CARVALHO JUNIOR, E. A. R.. Plano de Manejo Reserva Extrativista Tapajós-Arapiuns Santarém. Pará: ICMBIO, 2008.

CARVALHO, A. L.; NESSIMIAN, J. L.. Odonata do Estado do Rio de Janeiro, Brasil: habitats e hábitos das larvas. In: MARTINS, J. L.; CARVALHO, A. L.. Ecologia de Insetos Aquáticos. Rio de Janeiro: UFRJ, 1998. p.3-28.

CLAUSNITZER, V.. Dragonfly communities in coastal habitats of Kenya: indication of biotope quality and the need of conservation measures. Biodiversity and Conservation, v.12, p.333-356, 2003. DOI:

https://doi.org/10.1023/A:1021920402913

COBERT, P. S.; MAY, M. L.. Fliers and perchers among Odonata: dichotomy or multidimensional continuum. A provisional reappraisal. International Journal of Odonatology, v.11, n.2, p.155-171, 2008. DOI: https://doi.org/10.1080/13887890.2008.9748320

COBET, P. S.. Dragonflies: Behaviour and Ecology of Odonata. London: Comstock Publishing Association, 1999.

COLWELL, R. K.; CODDINGTON, J. A.. Estimating terrestrial biodiversity through extrapolation. Philosophical Transactions Royal Society London, Londres, v.345, n.1311, p.101-118, 1994. DOI:

https://doi.org/10.1098/rstb.1994.0091

COLWELL, R. K.; MAO, C. X.; CHANG, J.. Interpolatin, extrapolatin, and comparing incidence-based species accumulation curves. Ecology, v.85, p.2717-2727, 2004. DOI: https://doi.org/10.1890/03-0557

CONSATTI, G.; SANTOS, D. M.; RENNER, S.; PÉRICO E.. Presença de Odonata em áreas preservadas e não preservadas nas matas ciliares do rio Taquari, RS. Revista de Iniciação Científica da ULBRA, Canoas, n.12, p.57-65, 2014.

CÔRTES, L. G.; ALMEIDA, M. C.; PINTO, N. S.; MARCO, P.. Fogo em Veredas: Avaliação de impactos sobre comunidades de Odonata (Insecta). Revista Biodiversidade Brasileira, Brasília, v.1, n.2, p.128-145, 2011.

DAJOZ, R.. Ecologia Geral. 2 ed. Petrópolis: Vozes, 1973.
DALZOCHIO, M. S.; COSTA, J. M.; UCHÔA, M. A.. Diversity of Odonata (Insecta) in lotic systems from Serra da Bodoquena, Mato Grosso do Sul State, Brazil. Revista Brasileira de Entomologia, Curitiba, v.55, n.1, p.88-94, 2011. DOI: http://doi.org/10.1590/S0085-56262011000100014

DANTAS, T. A. V.. Diversidade de libélulas (Odonata: Libellulidae) em sistemas lênticos do brejo paraibano. Monografia (Graduação em Ciências Biológicas) - Universidade Federal da Paraíba, João Pessoa, 2017.

FERREIRA, A. C.; LIMA-FILHO, P. A.; LAYSE A. M.; ROCHA, E. $\mathrm{S}$.. A riqueza de Odonata difere em lagos permanentes e temporários no semiárido brasileiro?. Natal: 2009.

FERREIRA, D. A. C.; HERLY, C. T. D.. Situação atual da mata ciliar do ribeirão São Bartolomeu em Viçosa, MG. Revista Árvore, Viçosa, v.28, n.4, p.617-623, 2004.

FERREIRA-PERUQUETTI, P. S.; FONSECA-GESSNER, A. A.. Comunidade de Odonata (Insecta) em áreas naturais de Cerrado e monocultura no nordeste do Estado de São Paulo, Brasil: relação entre o uso do solo e a riqueza faunística. Revista Brasileira de Zoologia, Curitiba, v.20, p.219-224, 2003.

GARRISON, R. W.; ELLENRIEDER, N.; LOUTON, J. A. Damselfly Genera of the New World: an Illustrated and Annotated Key to the Zygoptera. Baltimore: The Johns Hopkins University Press, 2010.

GOULART, M.; CALLISTO, M.. Bioindicadores de qualidade de água como ferramenta em estudos de impacto ambiental. Revista FAPAM, Pará de Minas, v.2, n.2, p.153-164, 2003.

GUILHERMO-FERREIRA, R.; NEISS, U. G.; HAMADA, N.; BISPO, P. C.. Behavior of the Amazonian damselfly McLachlan (Zygoptera: Polythoridae) and comments on its morphological distinction from (Rambur). International Journal of Odonatology, v.17, p.1-8, 2014. DOI: https://doi.org/10.1080/13887890.2014.983189

HANAUER, G.; RENNER, S.; PÉRICO, E.. Inventariamento Preliminar da Fauna de libélulas (Odonata) em quatro municípios do vale do Taquari/RS. Revista Destaques Acadêmicos, Lajeado, v.6, n.3, p.36-45, 2014.

HURYN, A. D.; WALLACE, J. B.. Life History and Production of Stream Insects. Annual Reciew of Entomology, v.45, p.83110, 2000. DOI: https://doi.org/10.1146/annurev.ento.45.1.83

JANKOWSKI, J. E., CIECKA, A. L.; MEYER, N. Y.; RABENOLD, K. N.. Beta diversity along environmental gradients: implications of habitat specialization in tropical montane landscapes. Journal of Animal Ecology, v.78, n.2, p.315-327, 2009. DOI: https://doi.org/10.1111/j.1365-2656.2008.01487

JUEN, L.; MARCO, P.. Odonate beta diversity in terra-firme forest streams in Central Amazonia: On the relative effects of neutral and niche drivers at small geographical extents. Insect Conservation and Diversity, v.4, p.265-274, 2011. DOI: https://doi.org/10.1111/j.1752-4598.2010.00130.x

LOMOLINO, M. V.; PERAULT, D. R.. Geographic gradients of deforestation and mammalian communities in a fragmented, temperaterain forest landscape. Global Ecology and 
Biogeography, v.13, p.55-64, 2004. DOI: https://doi.org/10.1111/j.1466-882X.2004.00068.x

MACHADO, A. B. M.; MESQUITA, H. G.; MACHADO, P. A. R.. Contribuição ao conhecimento dos Odonatas da Estação Ecológica de Mereça. Acta Amazônica, Manaus, v.21, p.159173, 1991.

MARCO, P.. The Amazonian Campina dragonfly assemblage: patterns in microhabitat use and behavior in a foraging habitat (Anisoptera). Odonatologica, v.27, n.2, p.239-248, 1998.

MARCO, P.; COELHO, F. M.. Services performed by the ecosystem: forest remnants influence agricultural cultures' pollination and production. Biodiversity and Conservation, v.13, n.7 p.1245-1255, 2004. DOI: http://doi.org/10.1023/B:BIOC.0000019402.51193.e8

MARCO, P.; RESENDE, D. C.. Activity patterns and thermoregulation in a tropical dragonfly assemblage. Odonatologica, v.31, n.2, p.129-138, 2002.

MARCO, P.; VIANNA, D. M.. Distribuição do esforço de coleta de Odonata no Brasil: Subsídios para escolha de áreas prioritárias para levantamentos faunísticos. Lundiana, Belo Horizonte, n.6, p.13-26, 2005.

MARINS, A.; ROMANOWSKI, H. P.; MARCO, P.. Espécies de Odonata (Insecta) registradas no Parque Estadual de ItapuãViamão, RS, Brasil. In: CONGRESSO DE ECOLOGIA DO BRASIL. Anais. Caxambu: UFRGS, 2005.

MARTINS, E. G.. Comportamento territorial e reprodutivo de Argia sp. (Odonata: Coenagrionidae) em um igarapé da Amazônia Central. In: MACHADO, G.; MARCO, P.. Livro do curso de campo: Ecologia da Floresta Amazônica. Manaus: INPA, 2004.

MIGUEL, T. B.; CALVÃO, L. B.; VITAL, M. V. C.; JUEN, L. A.. Scientometric study of the order Odonata with special attention to Brazil. International Journal Of Odonatology. v.20, p.27-42, 2017. DOI: https://doi.org/10.1080/13887890.2017.1286267

MONTEIRO-JÚNIOR, C. S.. Efeitos da alteração ambiental causada pela urbanização na cidade de Manaus (AM) sobre comunidades de adultos de libélulas (Insecta: Odonata). Dissertação (Mestrado em Ciências Biológicas) - Instituto Nacional de Pesquisas da Amazônia, Manaus, 2012.
MONTEIRO-JÚNIOR, C. S.; ESPOSITO, M. C.; JUEN, L.. Are the adult odonate species found in a protected area different from those present in the surrounding zone? A case study from eastern Amazonia. Journal of Insect Conservation, v.20, n.4, p.643-652, 2016. DOI: https://doi.org/10.1007/s10841-016-9895-5

OLIVEIRA-JUNIOR, J. M. B.; CABETTE, H. S. R.; SILVA-PINTO, N.; JUEN, L.. As variações na comunidade de Odonata (Insecta) em córregos podem ser preditas pelo Paradoxo do Plâncton? Explicando a riqueza de espécies pela variabilidade ambiental. EntomoBrasilis, v.6, n.1, p.1-8, 2013.

OLIVEIRA-JUNIOR, J. M. B.; MARCO, P.; DIAS-SILVA, K.; LEITÃO, R. P.; LEAL, C. G.; POMPEU, P. S.; GARDER, T. A.; HUGHES, R. M.; JUEN. L.. Effects of human disturbance and riparian conditions on Odonata (Insecta) assemblages in eastern Amazon basin streams. Limnologica, v.66, p.31-39, 2017. DOI: https://doi.org/10.1016/j.limno.2017.04.007

OLIVEIRA-JUNIOR, J. M. B.; SHIMANO, Y.; GARDNER, T. A.; HUGHES, R. T. M.; MARCO JUNIOR., P.; JUEN, L.. Neotropical dragonflies (Insecta: Odonata) as indicators of ecological condition of small streams in the eastern Amazon. Austral Ecology, Austrália, v.40, n.6, p.733-744, 2015. DOI: https://doi.org/10.1111/aec.12242

SCHMIDT, E.. Habitat invitarization characterization and bioindication by a representative spectrum of Odonata species. Odonatologica, v.14, n.2 p.127-133, 1985.

SILVA, C. L.; KOKEN, A. F.; FURIERI, K. S.. Apoio à criação de unidades de conservação no Estado do Espírito Santo utilizando Odonata. In: CONGRESSO DE ECOLOGIA DO BRASIL, 8. Anais. Caxambu: UFMG, 2007.

SORIANO, E. G.. The first Mexican record of Perissolestes magdalenae (Williamson \& Williamson) (Zygoptera: Perilestidae). Notulae Odonatologicae, v.1, n.2, p.17-23, 1978.

SOUZA, L. O. I.; COSTA, J. M.; OLDRINI, B. B.. Odonata. In: FROEHLICH, C. G.. Guia on line: Identificação de larvas de Insetos Aquáticos do Estado de São Paulo. São Paulo: 2007.

ULRICH, W.. Species abundance distributions in space and time. Ecological Questions, v.9, p.5-20, 2008.

A CBPC - Companhia Brasileira de Produção Científica (CNPJ: 11.221.422/0001-03) detém os direitos materiais desta publicação. Os direitos referem-se à publicação do trabalho em qualquer parte do mundo, incluindo os direitos às renovações, expansões e disseminações da contribuição, bem como outros direitos subsidiários. Todos os trabalhos publicados eletronicamente poderão posteriormente ser publicados em coletâneas impressas sob coordenação da Sustenere Publishing, da Companhia Brasileira de Produção Científica e seus parceiros autorizados. Os (as) autores (as) preservam os direitos autorais, mas não têm permissão para a publicação da contribuição em outro meio, impresso ou digital, em português ou em tradução. 